\title{
Addition Reactions of 2-Amino-4-methoxypenta-2,4-dienenitrile with Electrophiles Containing Electron-deficient Multiple Bonds
}

\author{
Yu-Wen Wang, ${ }^{b}$ Jim-Min Fang, , ${ }^{*}$ Yu-Ker Wang, ${ }^{a}$ Mei-Huei Wang, ${ }^{a}$ Teng-Yao Ko ${ }^{a}$ and \\ Yie-Jia Chernga \\ a Department of Chemistry, National Taiwan University, Taipei, Taiwan 10764, Republic of China \\ b Department of Mathematics \& Physics Education, Taiwan Provincial Chiayi Teachers College, Taiwan. \\ Republic of China
}

\begin{abstract}
The title compound 1 underwent an insertion reaction with dichlorocarbene selectively at the C-4 double bond. The $[4+2]$ cycloadditions of 1 with benzoquinone and naphthoquinone proceeded by subsequent dehydrocyanation and oxidative aromatization under mild conditions to give the diarylamines 6 and 7. The cycloaddition of 1 and $\mathbf{N}$-phenylmaleimide in a sealed tube proceeded with reinsertion of the cyanide ion to give the diarylamine 8. The homologation of 1 with chlorosulfonyl isocyanate (CSI) and tetracyanoethylene (TCNE), giving the dienoate 9 and the triene 10, was unusual compared with commonly observed [2 + 2]cycloadditions of CSI and TCNE. The reaction of 1 with dimethyl acetylenedicarboxylate proceeded via an intermediate cyclobutene and tandem ring-opening afforded the triene 11. The reaction of 1 with ethyl propiolate produced two diarylamines $12 \mathrm{a}$ and $12 \mathrm{~b}$. Formation of $12 \mathrm{a}$ might involve cyanide reinsertion on the intermediate derived from the [4 +2$]$ cycloaddition, whereas an opening of the $[2+2]$ intermediate followed by an electrocyclization would yield the isomer $\mathbf{1 2 b}$.
\end{abstract}

Although the $\alpha$-aminoalkenenitriles have been used as acceptors in Michael reactions ${ }^{1}$ and as dienophiles in Diels-Alder reactions, ${ }^{2}$ we have reported that the related dienes $A$<smiles>[R]C=CC=C(C#N)N(C)c1ccccc1</smiles><smiles>[R]c1ccc(N(C)c2ccccc2)c2c1C(=O)OC2=O</smiles><smiles>[R]c1ccc2c(c1C(=O)O)c(=O)c1ccccc1n2[Y4]</smiles>

$(\mathrm{R}=\mathrm{Me}, \mathrm{Et}$ and $\mathrm{Ph}$ ) function as nucleophiles in cycloaddition with electron-deficient double bonds and in insertions with dichlorocarbene. ${ }^{3}$ The diarylamines $\mathbf{B}$, obtained by the reaction of $\mathbf{A}$ and maleic anhydride, are useful precursors for the acridones $\mathbf{C}$ formed by acid-catalysed cyclisations. We report here the reactions of 2-( $N$-methylanilino $)-4$-methoxypenta-2,4dienenitrile 1, whose reactivity toward electrophiles was

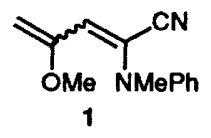

expected to be enhanced by the presence of the methoxy group. During the course of this study, we observed some unusual reactions of 1 owing to the effect of the methoxy group.

Results and Discussion

The allylic alcohol 2 was obtained in $90 \%$ yield by the reaction<smiles>C=C(O[C@H](O)C(C#N)N(C)c1ccccc1)[C@H](O)c1ccccc1</smiles><smiles>C=C(O[Na])/C(C)=C(/C#N)N(C)c1ccccc1</smiles><smiles>C=C(/C(=C(\C#N)Nc1ccccc1)C(C)C)N(C)c1ccccc1</smiles>

of the anion of 2-( $N$-methylanilino)acetonitrile with 2-methoxyacrylaldehyde. The methanesulfonate of the alcohol 2 was treated with $\mathrm{Bu}^{t} \mathrm{OK}$ to afford the desired diene 1 in $64 \%$ yield. Conversion of the allylic alcohol 2 to corresponding chloride $\left(\mathrm{POCl}_{3}\right.$, pyridine $)$ or acetate $\left(\mathrm{Ac}_{2} \mathrm{O}\right.$, pyridine), followed by elimination with various bases 1,4-diazabicyclo[2.2.2] octane, 1,5-diazabicyclo[4.3.0]non-5-ene, 1,8-diazabicyclo[5.4.0]undec-7-ene or $\left.\mathrm{Bu}^{\prime} \mathrm{OK}\right)$ gave lower yields of 1 . Unlike other stable $\alpha$-aminodienenitriles, compound 1 decomposed partially on a silica gel column, however, pure diene 1 was obtained by chromatography on neutral alumina. Although the $E$ - and $Z$ isomers of 1 were isolated for analytical purpose, the $E / Z$ mixture (predominating in the $Z$-isomer) was routinely used in subsequent reactions with a number of electrophiles. We also attempted to synthesise the diene 1 by using Peterson's method. ${ }^{3}$ The reaction did not yield the diene 1 , it gave instead the silylated derivative 3 accompanied by a self-condensation product 4.

Treatment of the diene 1 ( $Z$-configuration) with dichlorocarbene, generated from $\mathrm{CHCl}_{3}$ and $\mathrm{NaOH}$ by ultrasonication in the presence of a phase-transfer catalyst, ${ }^{4}$ yielded a cyclopropanation product 5 with the $Z$-configuration. However, the compound when dissolved in $\mathrm{CDCl}_{3}$ gradually became a mixture of the $E, Z$-isomers.

The reaction of 1 and p-benzoquinone occurred readily at room temperature to give a diarylamine 6 . This reaction probably involved a dipolar process to give a $[4+2]$ cycloaddition intermediate $D,{ }^{5}$ which subsequently lost HCN and underwent oxidative aromatization. ${ }^{3}$ Compared with the analogous reaction of the diene $\mathbf{A}$, which must be effected in refluxing xylene, the diene 1 appeared to be more reactive toward electrophiles. The similar reaction with 1,4-naphthoquinone also gave a high yield of the diarylamine 7 . When a benzene solution of the diene 1 and $N$-phenylmaleimide was 
<smiles>COC1(/C=C(/C#N)c2ccccc2)CC1(Cl)Cl</smiles>

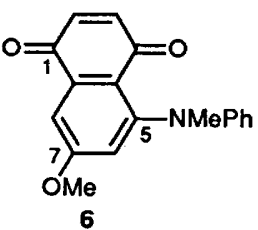

5<smiles></smiles>

7<smiles>COC1=CC(C)(Nc2ccccc2)C2C(=O)C=CC(=O)C2C1</smiles>

D<smiles>CN(c1ccccc1)c1cc(C#N)cc2c1C(=O)N(c1ccccc1)C2=O</smiles>

8

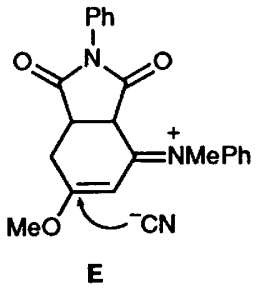

E

heated in a sealed tube, the diarylamine 8 with a cyano group replacing the methoxy substituent was obtained as the exclusive product. The characteristic IR absorption at $2235 \mathrm{~cm}^{-1}$ and the ${ }^{13} \mathrm{C}$ resonance at $\delta 117$ clearly indicated the presence of cyano group, and the parent ion at $\mathrm{m} / \mathrm{z} 353.115$ supported the structural assignment of $\mathbf{8}$. The reaction presumably involved counterattack of a cyanide ion on the conjugated iminium intermediate (E) followed by elimination of the methoxy group.

The reaction of diene 1 with chlorosulfonyl isocyanate CSI, followed by treatment with sodium methoxide, afforded the methyl dienoate 9. This result was in agreement with our<smiles>COC(=O)/C=C(\C=C(/C#N)Nc1ccccc1)OC</smiles><smiles>COC(C=C(C#N)Nc1ccccc1)=CC(C#N)=C(C#N)C#N</smiles><smiles>C=C(COC)/C(COC)=C(/C=C(\C#N)Nc1ccccc1)C(=O)OC</smiles>

previous report for homologation of the dienes $\mathbf{A}$ by adding to CSI instead of forming $\beta$-lactams. ${ }^{6}$ Compound 1 reacted readily with tetracyanoethylene TCNE in THF at $-78^{\circ} \mathrm{C}$ to give a quantitative amount of the triene 10. This reaction appeared to follow an addition-elimination mechanism. ? However, attempts to intercept the presumed zwitterion intermediate $\mathbf{F}^{8}$ with $\mathrm{MeOH}$ failed. The result was different<smiles>CO[C@H](/C=C(\C#N)Nc1ccccc1)CC(C#N)(C#N)C(C#N)C#N</smiles><smiles></smiles>

from the $[2+2]$ cycloadditions of the analogous dienes $\mathbf{A}$ with TCNE. $^{3,8}$ The reaction of 1 with dimethyl acetylenedicarboxylate in refluxing benzene gave an $88 \%$ yield of the triene 11 . By a DEPT technique, the ${ }^{13} \mathrm{C}$ resonance at $\delta 129.2$ was shown to be the olefinic methylene carbon $\left(\mathrm{H}_{2} \mathrm{C}=\right)$, it was presumed that the initially formed cyclobutene intermediate $\mathbf{G}$ readily underwent a ring rupture to give the thermodynamically stable product. $^{9}$

A toluene solution of $\mathbf{1}$ and ethyl propiolate in a sealed tube was refluxed for $16 \mathrm{~h}$ to give two diarylamines $12 \mathrm{a}(51 \%)$ and<smiles>CCOC(=O)c1ccc(C#N)cc1Nc1ccccc1</smiles>

$12 \mathrm{a}$<smiles>CCOC(=O)c1ccc(N(c2ccccc2)c2ccccc2)cc1C#N</smiles>

$12 b$
$12 \mathbf{b}(10 \%)$. The major compound 12a showed an IR absorption at $2227 \mathrm{~cm}^{-1}$ for the cyano group. The ${ }^{1} \mathrm{H}$ NMR spectrum revealed the absence of a methoxy group and the resonances of 5-H, 3-H and 6-H appeared at $\delta 6.84(\mathrm{dd}, J 9,3 \mathrm{~Hz}), 6.98(\mathrm{~d}, J 3$ $\mathrm{Hz})$ and $7.90(\mathrm{~d}, J 9 \mathrm{~Hz})$ as an $\mathrm{ABX}$ pattern. The ${ }^{13} \mathrm{C}$ resonances of 12a were in agreement with the theoretical values calculated for the assigned structure. ${ }^{10}$ The structure of $12 \mathrm{~b}$ was inferred from the ${ }^{1} \mathrm{H}$ and ${ }^{13} \mathrm{C}$ NMR spectrum, however, we were unable to obtain pure $12 \mathrm{~b}$ without contamination by 12a. Formation of 12a was considered to involve a cyanide ion counterattack on the iminium intermediate $\mathbf{H}$ as in the pathway proposed for<smiles>CCOC(=O)c1ccc(OC)cc1N=[P+]=N</smiles>

H<smiles>[Y]C1(/C=C(\C#N)Nc2ccccc2)CC=C1C(=O)OC</smiles>

I $(Y=O M e$ or $C N)$<smiles>[Y]C(/C=C(/C#N)Nc1ccccc1)=C(/C=C)C(=O)OCC</smiles>

$J(Y=O M e$ or $C N)$

compound 8. On the other hand, ring opening of the cyclobutene intermediate I might give $\mathbf{J},{ }^{9}$ and subsequent electrocyclization would eventually lead to product $12 \mathrm{~b} .{ }^{11}$

In summary, the $\alpha$-aminodienenitrile 1 having a methoxy group at $\mathrm{C}-4$ exerted remarkable nucleophilicity toward various substrates containing double or triple bonds. Compound 1 followed the similar pathway of analogous $\alpha$-amino dienenitriles $A$ in reactions with $p$-benzoquinone, 1,4-naphthoquinone, dichlorocarbene and chlorosulfonyl isocyanate, while 1 reacted unusually with TCNE and dimethyl acetylenedicarboxylate. It was noted that the reactions with $\mathrm{N}$ phenylmaleimide and ethyl propiolate in sealed tubes caused counterattack of the cyanide ion to replace the methoxy group.

\section{Experimental}

M.p.s are not corrected. ${ }^{1} \mathrm{H}$ NMR spectra were recorded at 200 or $300 \mathrm{MHz}$ while ${ }^{13} \mathrm{C}$ NMR recorded at 50 or $75 \mathrm{MHz}(J$ values in $\mathrm{Hz}$ ). Mass spectra were recorded at an ionizing voltage of $70 \mathrm{eV}$. Merck silica gel 60F sheets were used for analytical TLC. HPLC was performed on a $\mu$-Porasil column $(0.78$ $\times 25 \mathrm{~cm})$ using the indicated eluent with $5 \mathrm{~cm}^{3} \mathrm{~min}^{-1}$ flow rate. $2-(N$-Methylanilino)acetonitrile was prepared by the Strecker's method. ${ }^{12}$ 2-Methoxyacrylaldehyde was prepared from formaldehyde and 2-methoxyacetaldehyde. ${ }^{13}$

4-Methoxy-2-(N-methylanilino)penta-2,4-dienenitrile 1.Under an atmosphere of nitrogen, BuLi $\left(1.6 \mathrm{~mol} \mathrm{dm}^{-3}\right.$ solution in hexane; $0.69 \mathrm{~cm}^{3}, 1.1 \mathrm{mmol}$ ) was added dropwise to a solution 
of diisopropylamine $\left(0.17 \mathrm{~cm}^{3}, 1.2 \mathrm{mmol}\right)$ in tetrahydrofuran (THF) $\left(2 \mathrm{~cm}^{3}\right)$ at $-10^{\circ} \mathrm{C}$. The solution was stirred for $10 \mathrm{~min}$, and a solution of $2-(N$-methylanilino $)$ acetonitrile $(153 \mathrm{mg}, 1.05$ $\mathrm{mmol})$ in THF $\left(1 \mathrm{~cm}^{3}\right)$ was added dropwise. After $15 \mathrm{~min}$, the solution was cooled to $-78^{\circ} \mathrm{C}$, and a solution of 2 methoxyacrylaldehyde $(126 \mathrm{mg}, 1.05 \mathrm{mmol})$ in THF $\left(0.5 \mathrm{~cm}^{3}\right)$ was added dropwise. The reaction mixture was warmed to room temp. and quenched by addition of acetic acid $\left(1.2 \mathrm{~cm}^{3}\right)$. The volatile components were removed under reduced pressure and the residue was partitioned between EtOAc and water. The aqueous phase was extracted $(2 \times$ EtOAc). The combined organic phase was washed with brine, dried $\left(\mathrm{Na}_{2} \mathrm{SO}_{4}\right)$, and concentrated to give the allylic alcohol $2(220 \mathrm{mg}, 0.95 \mathrm{mmol})$ (two diastereoisomers). A mixture of the alcohol $2(220 \mathrm{mg})$ and triethylamine $\left(0.3 \mathrm{~cm}^{3}\right)$ in $\mathrm{CH}_{2} \mathrm{Cl}_{2}\left(2 \mathrm{~cm}^{3}\right)$ was treated with methanesulfonyl chloride $\left(0.1 \mathrm{~cm}^{3}, 1.3 \mathrm{mmol}\right)$ at $0{ }^{\circ} \mathrm{C}$. The reaction mixture was slowly warmed to room temp. over a period of $2 \mathrm{~h}$ and then heated to $40^{\circ} \mathrm{C}$ for $16 \mathrm{~h}$. An $\mathrm{HCl}$ solution $(5 \%)$ was added, and the mixture was extracted $(3 \times$ EtOAc). The combined extracts were washed with brine, dried and concentrated to give the corresponding methanesulfonate (284 $\mathrm{mg})$. To a THF solution $\left(5 \mathrm{~cm}^{3}\right)$ of ButOK $(110 \mathrm{mg}, 1 \mathrm{mmol})$ cooled to $-78{ }^{\circ} \mathrm{C}$, was added dropwise a THF solution $\left(1 \mathrm{~cm}^{3}\right)$ of the above prepared methanesulfonate. The mixture was stirred for $30 \mathrm{~min}$, and then a solution of $\mathrm{AcOH}(3 \mathrm{mmol})$ in THF was added. The mixture was concentrated under reduced pressure, and the residue was chromatographed on $\mathrm{Al}_{2} \mathrm{O}_{3} 90$ (neutral, activity I) by elution with $5 \%$ EtOAc in hexane to give the diene 1 (134 $\mathrm{mg}, 60 \%$ overall yield).

$Z$-Isomer (major): yellow oil; $v_{\max }$ (neat) $/ \mathrm{cm}^{-1}$ 2949, 2214 $(\mathrm{CN}), 1595,1362,1192,824$ and $754 ; \delta_{\mathrm{H}}\left(\mathrm{CDCl}_{3} ; 300 \mathrm{MHz}\right)$ 3.14 ( $3 \mathrm{H}, \mathrm{s}, \mathrm{NMe}), 3.32$ ( $3 \mathrm{H}, \mathrm{s}, \mathrm{OMe}), 4.34(1 \mathrm{H}, \mathrm{d}, J 2.6,5-\mathrm{H})$, $4.43(1 \mathrm{H}, \mathrm{d}, J 2.6,5-\mathrm{H}), 6.12(1 \mathrm{H}, \mathrm{s}, 3-\mathrm{H}), 6.62(2 \mathrm{H}, \mathrm{m}), 6.69(1$ $\mathrm{H}, \mathrm{m})$ and $7.27(2 \mathrm{H}, \mathrm{m}) ; \delta_{\mathrm{C}}\left(\mathrm{CDCl}_{3} ; 75 \mathrm{MHz}\right) 38.7(\mathrm{NMe})$ 54.8 (OMe), 91.6 (C-5), 115.7 (2 C, C-2', C-6'), $117.0(\mathrm{CN}), 118.9$

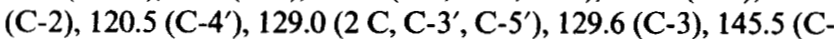
$\left.1^{\prime}\right)$ and $156.1(\mathrm{C}-4) ; m / z 214\left(\mathrm{M}^{+}, 67 \%\right), 213\left(\mathrm{M}^{+}-1,100\right), 199$ $\left(\mathbf{M}^{+}-15,75\right), 172(19)$ and $77(68)\left(M^{+}, 214.1114 . M\right.$, 214.1106).

$E$-Isomer: yellow oil; $v_{\max }$ (neat) $/ \mathrm{cm}^{-1} 2950,2210$ (CN), $1595,1360,1160,840$ and $770 ; \delta_{\mathrm{H}}\left(\mathrm{CDCl}_{3} ; 300 \mathrm{MHz}\right) 3.11(3$ $\mathrm{H}, \mathrm{s}, \mathrm{NMe}), 3.38$ (3 H, s, OMe), 4.09 (1 H, s, 3-H), 4.50 (1 H, d, $J$ $2.5), 4.59(1 \mathrm{H}, \mathrm{d}, J 2.5), 7.11(2 \mathrm{H}, \mathrm{m}), 7.15(1 \mathrm{H}, \mathrm{m})$ and $7.27(2$ $\mathrm{H}, \mathrm{m}) ; m / z 214\left(\mathrm{M}^{+}, 77^{\circ}\right), 213\left(\mathrm{M}^{+}-1,100\right), 199\left(\mathrm{M}^{+}-15\right.$, 62), $172(20), 144(19), 106(14), 82(24)$ and $77(69)\left(\mathrm{M}^{+}\right.$, 214.1114. $M, 214.1106)$.

\section{4-Methoxy-2-(N-methylanilino)-3-trimethylsilylpenta-2,4-} dienenitrile 3 and 2,4-Bis(N-methylanilino)-3-[bis(trimethylsilyl)amino]but-2-enenitrile 4.-A lithium diisopropylamide (LDA) solution $(11 \mathrm{mmol})$ was prepared at $-10^{\circ} \mathrm{C}$, and a solution of $2-(N$-methylanilino)acetonitrile $(1.46 \mathrm{~g}, 10 \mathrm{mmol})$ in THF $\left(7 \mathrm{~cm}^{3}\right)$ was added dropwise. The solution was stirred for $30 \mathrm{~min}$, cooled to $-78^{\circ} \mathrm{C}$, and chlorotrimethylsilane $\left(1.6 \mathrm{~cm}^{3}\right.$, $10 \mathrm{mmol}$ ) was added dropwise. The mixture was stirred for 30 min after which additional LDA solution $(10 \mathrm{mmol})$ was added. Afte: $1 \mathrm{~h}$, a solution of 2-methoxyacrylaldehyde $(1.23 \mathrm{~g}, 10$ $\mathrm{mmol})$ in THF $\left(5 \mathrm{~cm}^{3}\right)$ was added dropwise. The reaction mixture was warmed to room temp. for $16 \mathrm{~h}$, and then quenched with saturated aqueous $\mathrm{NH}_{4} \mathrm{Cl}\left(15 \mathrm{~cm}^{3}\right)$. The volatiles were removed under reduced pressure and the residue was extracted $(3 \times$ EtOAc). The combined extracts were washed with brine, dried and concentrated to give a crude oil, which was chromatographed on a $\mathrm{SiO}_{2}$ column by elution with $2 \%$ EtOAc in hexane to afford the silylated diene $3,(1.21 \mathrm{~g}, 43 \%)$, as a mixture of $E$ - and $Z$-isomers $(3: 1)$ and a side-product 4 $(0.65 \mathrm{~g})$.

$E$-Isomer of 3: colourless crystals, m.p. $60-61^{\circ} \mathrm{C}$; HPLC $(2 \%$
EtOAc in hexane) $t_{\mathrm{R}} 4.9 \mathrm{~min} ; v_{\max }(\mathrm{KBr}) / \mathrm{cm}^{-1} 2952,2213$, $1637,1596,1494,1191,995,845$ and $753 ; \delta_{\mathrm{H}}\left(\mathrm{CDCl}_{3}\right) 0.34(9$ $\mathrm{H}, \mathrm{s}), 3.12(3 \mathrm{H}, \mathrm{s}), 3.42(3 \mathrm{H}, \mathrm{s}), 3.84(1 \mathrm{H}, \mathrm{d}, J 2.7), 3.96(1 \mathrm{H}, \mathrm{d}$, $J$ 2.7), $6.80(2 \mathrm{H}, \mathrm{m}), 6.89(1 \mathrm{H}, \mathrm{m})$ and $7.27(2 \mathrm{H}, \mathrm{m})$; $\delta_{\mathrm{C}}\left(\mathrm{CDCl}_{3}\right)-1.3\left(\mathrm{q}, 3 \mathrm{C}, \mathrm{Me}_{3} \mathrm{Si}\right), 38.9(\mathrm{q}, \mathrm{NMe}), 54.6(\mathrm{q}$, $\mathrm{OMe}$ ), 82.5 (t, C-5), 116.2 (d, $2 \mathrm{C}$ ), 116.6 (s, CN), 120.4 (d), 126.6 (s, C-3), 129.0 (2 C), 145.8 (s, C-2), 147.4 (s) and 159.0 (s, C-4); $m / z 286\left(\mathrm{M}^{+}, 17 \%\right), 271(56), 181(24), 167(22), 89(27)$ and 77 (100) (Found: $\mathrm{C}, 67.1 ; \mathrm{H}, 7.8 ; \mathrm{N}, 9.9) . \mathrm{C}_{16} \mathrm{H}_{22} \mathrm{~N}_{2} \mathrm{OSi}$ requires $\mathrm{C}$, $67.09 ; 7.74 ; \mathrm{N}, 9.78 \%$.

$Z$-Isomer of 3: colourless crystals, m.p. $71-72{ }^{\circ} \mathrm{C}$; HPLC $(2 \%$ EtOAc in hexane) $t_{\mathrm{R}} 6.8 \mathrm{~min} ; v_{\max }(\mathrm{KBr}) / \mathrm{cm}^{-1} 2955,2200$, $1595,1491,1154,994,846$ and $757 ; \delta_{\mathrm{H}}\left(\mathrm{CDCl}_{3}\right) 0.09(9 \mathrm{H}, \mathrm{s})$, $3.04(3 \mathrm{H}, \mathrm{s}), 3.63(3 \mathrm{H}, \mathrm{s}), 4.27(1 \mathrm{H}, \mathrm{d}, J 2.7), 4.32(1 \mathrm{H}, \mathrm{d}, J 2.7)$, $6.80(2 \mathrm{H}, \mathrm{m}), 6.90(1 \mathrm{H}, \mathrm{m})$ and $7.27(2 \mathrm{H}, \mathrm{m}) ; \delta_{\mathrm{C}}\left(\mathrm{CDCl}_{3}\right)$ $-1.2\left(\mathrm{q}, 3 \mathrm{C}, \mathrm{Me}_{3} \mathrm{Si}\right), 39.4$ (q, NMe), 55.1 (q, OMe), 85.1 (t, C-5), 114.2 (s, CN), 114.8 (d, 2 C), 120.3 (d), 127.9 (s, C-3), 129.1 (s), $129.2(2 \mathrm{C}), 147.1 \mathrm{~s}$ ) and $160.5(\mathrm{~s}, \mathrm{C}-4) ; \mathrm{m} / \mathrm{z} 286\left(\mathrm{M}^{+}, 14 \%\right), 271$ (51), 181 (20), 158 (22) and 77 (100) (Found: C, 67.0; H, 7.8; N, 10.0. $\mathrm{C}_{16} \mathrm{H}_{22} \mathrm{~N}_{2}$ OSi requires $\mathrm{C}, 67.09 ; \mathrm{H}, 7.74 ; \mathrm{N}, 9.78 \%$ ).

Nitrile 4: Colourless platelets, m.p. 97.5-98.5 ${ }^{\circ} \mathrm{C}$ (hexane); $v_{\max }(\mathrm{KBr}) / \mathrm{cm}^{-1} 2954,2204,1598,1498,1256,902$ and 843; $\delta_{\mathrm{H}}\left(\mathrm{CDCl}_{3}\right) 0.30\left(18 \mathrm{H}, \mathrm{s}, 2 \times \mathrm{Me}_{3} \mathrm{Si}\right), 2.91(3 \mathrm{H}, \mathrm{s}, \mathrm{NMe}), 3.13$ $(3 \mathrm{H}, \mathrm{s}, \mathrm{NMe}), 3.98(2 \mathrm{H}, \mathrm{s})$ and $6.67-7.30(10 \mathrm{H}) ; \delta_{\mathrm{C}}\left(\mathrm{CDCl}_{3}\right)$ $2.88\left(\mathrm{Me}_{3} \mathrm{Si}\right), 39.8(\mathrm{NMe}), 40.5(\mathrm{NMe}), 55.8\left(\mathrm{CH}_{2}\right), 112.2(\mathrm{~s})$, 112.7 (d), 114.6 (d), 116.1 (s), 116.3 (s), 116.9 (d), 118.7 (s), 120.0 (d), 128.8 (d), 129.2 (d), 147.1 (s) and 149.8 (s); $\mathrm{m} / \mathrm{z} 436\left(\mathrm{M}^{+}\right.$, $36 \%$ ), 421 (5), $330(90), 300(22), 275$ (12), 274 (15) and $120(110)$ (Found: $\mathrm{C}, 65.9 ; \mathrm{H}, 8.2 ; \mathrm{N}, 12.95 . \mathrm{C}_{24} \mathrm{H}_{36} \mathrm{~N}_{4} \mathrm{Si}_{2}$ requires $\mathrm{C}, 66.0$; $\mathrm{H}, 8.31 ; \mathrm{N}, 12.83 \%$ ).

3-(2,2-Dichloro-1-methoxycyclopropyl)-2-(N-methylanilino)prop-2-enenitrile 5.-To a mixture of the diene 1 ( $Z$-isomer; 178 $\mathrm{mg}, 0.83 \mathrm{mmol})$ and benzyltriethylammonium chloride $(5 \mathrm{mg})$ in $\mathrm{CHCl}_{3}\left(2 \mathrm{~cm}^{3}\right)$ was added dropwise aqueous $\mathrm{KOH}(60 \% ; 1$ $\mathrm{cm}^{3}$ ) at $0{ }^{\circ} \mathrm{C}$. The viscous mixture was sonicated for $3.5 \mathrm{~h}$ while the temperature was kept at $0-5{ }^{\circ} \mathrm{C}$. The mixture was diluted with water $\left(5 \mathrm{~cm}^{3}\right)$ and extracted $\left(3 \times \mathrm{CHCl}_{3}\right)$. The combined organic phase was dried, concentrated, and purified by a short silica gel column by elution with EtOAc to give compound 5 , (232 $\mathrm{mg}, 0.78 \mathrm{mmol}, 94 \%$ ), ( $Z$-form) which became the $E / Z$ mixture with time.

$Z$-Isomer: $v_{\max }$ (neat) $/ \mathrm{cm}^{-1}$ 2933, 2233, 1591, 1493, 1351, $1250,1007,855$ and $699 ; \delta_{\mathrm{H}}\left(\mathrm{CDCl}_{3}\right) 1.78\left(1 \mathrm{H}, \mathrm{d}, J 11.3,3^{\prime}-\mathrm{H}\right)$, 1.77 ( $\left.1 \mathrm{H}, \mathrm{d}, J 11.3,3^{\prime}-\mathrm{H}\right), 3.32$ ( $\left.3 \mathrm{H}, \mathrm{s}, \mathrm{NMe}\right), 3.35$ (3 H, s, OMe), $5.85(1 \mathrm{H}, \mathrm{s}, 3-\mathrm{H}), 6.96(3 \mathrm{H}, \mathrm{m})$ and $7.34(2 \mathrm{H}, \mathrm{m}) ; \delta_{\mathrm{C}}\left(\mathrm{CDCl}_{3}\right)$ 34.1 (q, NMe), $39.2\left(\mathrm{t}, \mathrm{C}-3^{\prime}\right), 55.9(\mathrm{q}, \mathrm{OMe}), 63.8\left(\mathrm{~s}, \mathrm{C}-2^{\prime}\right), 64.8(\mathrm{~s}$, $\left.\mathrm{C}-1^{\prime}\right), 115.7$ (s, CN), 117.2 (d, 2 C, C-2", C-6"), 121.8 (d, C-3), 124.4 (s, C-2), 125.2 (d, C-4"), 129.1 (d, 2 C, C-3", C-5") and 144.7 (s, C-1"); m/z $297\left(\mathrm{M}^{+}+1,9 \%\right), 296\left[\mathrm{M}^{+}\left({ }^{35} \mathrm{Cl}\right), 8 \%\right], 262$ (34), 261 (86), 260 (30), 225 (100), $210(9), 199$ (13), 185 (48), 142 (23), $107(47)$ and $77(79)\left(\mathrm{M}^{+}, 296.0466 . M, 296.0483\right)$.

$E$-Isomer: $\delta_{\mathrm{H}}\left(\mathrm{CDCl}_{3}\right) 1.95(1 \mathrm{H}, \mathrm{d}, J 13.5), 2.01(1 \mathrm{H}, \mathrm{d}, J$ 13.5), 3.22 (3 H, s, NMe), 3.46 ( $3 \mathrm{H}, \mathrm{s}, \mathrm{OMe}), 5.58(1 \mathrm{H}, \mathrm{s}, 3-\mathrm{H})$, $7.16(3 \mathrm{H}, \mathrm{m})$ and $7.37(2 \mathrm{H}, \mathrm{m}) ; \delta_{\mathrm{C}}\left(\mathrm{CDCl}_{3}\right) 33.2(\mathrm{q}, \mathrm{NMe})$, $40.6\left(\mathrm{t}, \mathrm{C}-3^{\prime}\right), 55.1$ (q, OMe), $63.8\left(\mathrm{~s}, \mathrm{C}-2^{\prime}\right), 65.0\left(\mathrm{~s}, \mathrm{C}-1^{\prime}\right), 111.2$ (d, C-3), 113.4 (s, CN), 122.0 (s, C-2), 124.1 (d, 2 C, C-2", C-6"), 125.8 (d, C-4"), 129.4 (d, 2 C, C-3", 5") and 145.2 (s, C-1").

7-Methoxy-5-(N-methylanilino)-1,4-naphthoquinone 6- - $\mathrm{A}$ benzene solution $\left(3 \mathrm{~cm}^{3}\right)$ of the diene $1(E / Z$ mixture; $60 \mathrm{mg}$, $0.28 \mathrm{mmol}$ ) and $p$-benzoquinone ( $40 \mathrm{mg}, 0.37 \mathrm{mmol}$ ) was stirred at room temp. for $16 \mathrm{~h}$. The solvent was removed, and the residue was chromatographed on $\mathrm{SiO}_{2}$ by elution with gradients of EtOAc in hexane to give compound $6(74 \mathrm{mg}, 90 \%)$, as purple crystals, m.p. $99-101{ }^{\circ} \mathrm{C}$; TLC $(5 \%$ EtOAc in hexane) $R_{\mathrm{f}} 0.14 ; v_{\max }(\mathrm{KBr}) / \mathrm{cm}^{-1} 2938,1660(\mathrm{C}=\mathrm{O}), 1583,1464,1268$, $1184,1085,990,845$ and $749 ; \delta_{\mathrm{H}}\left(\mathrm{CDCl}_{3} ; 300 \mathrm{MHz}\right) 3.25(3 \mathrm{H}$, 
s, NMe), $3.89(3 \mathrm{H}, \mathrm{s}, \mathrm{OMe}), 6.75(5 \mathrm{H}, \mathrm{m}), 7.07(1 \mathrm{H}, \mathrm{d}, J 2.9)$, $7.19(2 \mathrm{H}, \mathrm{m})$ and $7.47(1 \mathrm{H}, \mathrm{d}, J 2.9) ; \delta_{\mathrm{C}}\left(\mathrm{CDCl}_{3} ; 75 \mathrm{MHz}\right)$, 40.3 (q, NMe), 55.9 (q, OMe), 109.2 (d, C-6), 115.5 (d, 2 C, C-2', C-6'), 119.2 (d, C-8), 120.1 (d, C-4'), 129.0 (d, 2 C, C-3', C-5'), 135.8 (d, C-3), 136.6 (s, C-5), 141.2 (d, C-2), 148.4 (s, C-10), 150.9 (s, C-9), $164.0(\mathrm{~s}, \mathrm{C}-7), 182.0(\mathrm{~s}, \mathrm{C}=\mathrm{O})$ and $185.2(\mathrm{~s}, \mathrm{C}=\mathrm{O}) ; \mathrm{m} / \mathrm{z}$ $293\left(\mathrm{M}^{+}, 80 \%\right), 276(100)$ and 261 (28) (Found: C, 73.3; H, 5.2; $\mathrm{N}, 4.9 . \mathrm{C}_{18} \mathrm{H}_{15} \mathrm{NO}_{3}$ requires $\mathrm{C}, 73.71 ; \mathrm{H}, 5.15 ; \mathrm{N}, 4.78 \%$ ).

3-Methoxy-1-(N-methylanilino)-9,10-anthraquinone 7.-A benzene solution $\left(10 \mathrm{~cm}^{3}\right)$ of the diene $1(E / Z$ mixture, $489 \mathrm{mg}$, $2.3 \mathrm{mmol}$ ) and 1,4-naphthoquinone (370 $\mathrm{mg}, 2.4 \mathrm{mmol})$ was heated at $60^{\circ} \mathrm{C}$ for $16 \mathrm{~h}$. The solvent was removed, and the residue was chromatographed on $\mathrm{SiO}_{2}$ by elution with gradients of EtOAc in hexane to give compound 7, $(641 \mathrm{mg}$, $82 \%$ ), as purple crystals, m.p. $197-198{ }^{\circ} \mathrm{C}$; TLC $(1 \%$ EtOAc in hexane) $R_{\mathrm{f}} 0.21 ; v_{\max }(\mathrm{KBr}) / \mathrm{cm}^{-1} 3441,1731 \mathrm{w}, 1667 \mathrm{~s}, 1584$, 1300 and $884 ; \delta_{\mathrm{H}}\left(\mathrm{CDCl}_{3} ; 300 \mathrm{MHz}\right) 3.31(3 \mathrm{H}, \mathrm{s}, \mathrm{NMe}), 3.93$ (3 H, s, OMe), $6.76(3 \mathrm{H}, \mathrm{m}), 7.16(3 \mathrm{H}, \mathrm{m}), 7.69(3 \mathrm{H}, \mathrm{m}), 8.11(1$ $\mathrm{H}, \mathrm{m})$ and $8.21(1 \mathrm{H}, \mathrm{m}) ; \delta_{\mathrm{C}}\left(\mathrm{CDCl}_{3} ; 75 \mathrm{MHz}\right) 40.2(\mathrm{q}, \mathrm{NMe})$, 55.9 (q, OMe), 109.6 (d, C-2), 114.9 (d, 2 C, C-2', C-6'), 118.7 (d, C-4), 121.3 (d, C-4'), 126.6 (d, C-5), 127.2 (d, C-8), 129.0 (d, 2 C, C-3', C-5'), 133.0 (d, C-6), 134.3 (d, C-7), 135.0 (s, C-1'), 138.2 (s, C-1), 148.5 (s, 2 C, C-11, C-12), 151.4 (s, 2 C, C-13, C-14), 164.1 (s, C-3), $180.3(\mathrm{~s}, \mathrm{C}=\mathrm{O})$ and $183.4(\mathrm{~s}, \mathrm{C}=\mathrm{O}) ; \mathrm{m} / \mathrm{z} 343\left(\mathrm{M}^{+}\right.$, 90\%), 326 (100), 251 (16), $106(12)$ and 77 (10) (Found: C, $76.4 ; \mathrm{H}, 4.95 ; \mathrm{N}, 3.8 . \mathrm{C}_{22} \mathrm{H}_{17} \mathrm{NO}_{3}$ requires $\mathrm{C}$, 76.95; $\mathrm{H}, 4.99$; $\mathrm{N}, 4.08 \%$ ).

6-Cyano-4-(N-methylanilino)-N-phenylphthalimide 8.-A benzene solution $\left(5 \mathrm{~cm}^{3}\right)$ of the diene $1(E / Z$ mixture, $326 \mathrm{mg}$, $1.5 \mathrm{mmol})$ and $N$-phenylmaleimide $(263 \mathrm{mg}, 1.5 \mathrm{mmol})$ was refluxed at $80^{\circ} \mathrm{C}$ in a sealed tube for $16 \mathrm{~h}$. The solvent was removed, and the residue was chromatographed on $\mathrm{SiO}_{2}$ by elution with gradients of EtOAc in hexane to give compound 8 $(386 \mathrm{mg}, 72 \%)$, as orange crystals, m.p. $164-165^{\circ} \mathrm{C}$; HPLC $\left(20 \%\right.$ EtOAc in hexane) $t_{\mathrm{R}} 5.8 \mathrm{~min} ; v_{\max }(\mathrm{KBr}) / \mathrm{cm}^{-1} 2235(\mathrm{CN})$, $1770,1720(\mathrm{C}=\mathrm{O}), 1571,1493,1127,979,842$ and $754 ; \delta_{\mathrm{H}}$ $\left(\mathrm{CDCl}_{3} ; 300 \mathrm{MHz}\right) 3.57(3 \mathrm{H}, \mathrm{s}, \mathrm{NMe}), 7.08-7.17(3 \mathrm{H}, \mathrm{m}), 7.32-$ $7.39(5 \mathrm{H}, \mathrm{m}), 7.44-7.51(2 \mathrm{H}, \mathrm{m}), 7.54(1 \mathrm{H}, \mathrm{s}, 4-\mathrm{H})$ and $7.68(1$ $\mathrm{H}, \mathrm{s}, 7-\mathrm{H}) ; \delta_{\mathrm{C}}\left(\mathrm{CDCl}_{3} ; 75 \mathrm{MHz}\right) 42.7$ (q, NMe), $117.0(\mathrm{~s}, \mathrm{CN})$, 118.2 (d, C-5), 121.0 (s, C-6), 121.8 (d, 2 C, C-2", C-6"), 124.6 (d), 126.6 (d, 2 C, C-2', C-6'), 128.4 (d), 129.2 (d, 2 C, C-3", C-5"), 129.9 (d, 2 C, C-3' C- $\left.5^{\prime}\right), 131.3$ (s, C-1') 132.4 (d, C-7), 135.5 (s, C4), 147.1 (s, C-9), $148.2(\mathrm{~s}, \mathrm{C}-8), 164.5(\mathrm{~s}, \mathrm{C}=\mathrm{O})$ and $165.3(\mathrm{~s}$ $\mathrm{C}=\mathrm{O}) ; \mathrm{m} / \mathrm{z} 353\left(\mathrm{M}^{+}, 100^{\%}\right), 324(7), 308(10), 276(15), 205$ (14), 177 (18), $106(21), 77$ (35), $\left(\mathrm{M}^{+}, 353.1147 . M, 353.1164\right)$.

Methyl 5-Cyano-3-methoxy-5-(N-methylanilino)penta-2,4-dienoate 9.- To a solution of the diene 1 ( $E / Z$ mixtue, $892 \mathrm{mg}, 4.2$ $\mathrm{mmol})$ in $\mathrm{CH}_{2} \mathrm{Cl}_{2}\left(10 \mathrm{~cm}^{3}\right)$ at $-78^{\circ} \mathrm{C}$ was added chlorosulfonyl isocyanate $\left(0.4 \mathrm{~cm}^{3}, 4.5 \mathrm{mmol}\right)$. The mixture was stirred for $1 \mathrm{~h}$, after which sodium methoxide $(675 \mathrm{mg}, 12.5 \mathrm{mmol})$ was added and stirring continued for a further $15 \mathrm{~min}$. After addition of $\mathrm{MeOH}\left(5 \mathrm{~cm}^{3}\right)$, the reaction mixture was concentrated under reduced pressure, and the residue was separated by chromatography on $\mathrm{SiO}_{2}$ column by elution with gradients of EtOAc in hexane to give the ester $9(613 \mathrm{mg}, 54 \%)$, as a brownish-yellow oil; TLC ( $2 \%$ EtOAc in hexane) $R_{\mathrm{f}} 0.27 ; v_{\max }$ (neat) $/ \mathrm{cm}^{-1} 3180$, $2943,2229,1710,1688,1510,1320$ and $870 ; \delta_{\mathrm{H}}\left(\mathrm{CDCl}_{3} ; 300\right.$ $\mathrm{MHz}) 3.30$ (3 H, s, NMe), 3.66 (3 H, s, OMe), 3.73 ( $3 \mathrm{H}, \mathrm{s}, \mathrm{OMe}$ ), $5.04(1 \mathrm{H}, \mathrm{s}, 2-\mathrm{H}), 7.18(1 \mathrm{H}, \mathrm{s}, 4-\mathrm{H}), 7.23(3 \mathrm{H}, \mathrm{m})$ and $7.34(2 \mathrm{H}$, $\mathrm{m}) ; \delta_{\mathrm{C}}\left(\mathrm{CDCl}_{3} ; 75 \mathrm{MHz}\right) 41.5(\mathrm{q}, \mathrm{NMe}), 50.9$ (q, OMe), 55.2 (q, OMe), 90.0 (d, C-2), 107.8 (d, C-4), 114.0 (s, CN), 124.6 (s, C5), 125.5 (d, 2 C, C-2', C-6'), 126.6 (d, C-4'), 129.4 (d, 2 C, C-3', C-5'), 145.3 (s, C-1'), 165.4 (s, C-3) and $167.9(\mathrm{~s}, \mathrm{C}=\mathrm{O}) ; m / 2272$ $\left(\mathrm{M}^{+}, 48 \%\right), 271(64), 225(33), 213(100), 169(14), 128(15)$ and 77 (27). ( $\left.\mathrm{M}^{+}, 272.1143 . M, 272.1161\right)$.
4-Methoxy-6-(N-methylanilino)hexa-1,3,5-triene-1,1,2,6tetracarbonitrile 10.-A THF solution $\left(5 \mathrm{~cm}^{3}\right)$ of the diene 1 $(E / Z$ mixture, $96 \mathrm{mg}, 0.45 \mathrm{mmol})$ and tetracyanoethylene $(58$ $\mathrm{mg}, 0.45 \mathrm{mmol}$ ) was stirred at $-78^{\circ} \mathrm{C}$ for $1.5 \mathrm{~h}$. The solvent was removed, and the residue was chromatographed on $\mathrm{SiO}_{2}$ by elution with gradients of EtOAc in hexane to give the triene 10 (130 mg, 92\%), as a purple oil; TLC (15\% EtOAc in hexane) $R_{\mathrm{f}}$ $0.13 ; v_{\max }($ neat $) / \mathrm{cm}^{-1} 2946,2212,1526,1487,1307,1132,768$ and $699 ; \delta_{\mathrm{H}}\left(\mathrm{CDCl}_{3} ; 300 \mathrm{MHz}\right) 3.50(3 \mathrm{H}, \mathrm{s}, \mathrm{NMe}), 3.94(3 \mathrm{H}, \mathrm{s}$, OMe), 5.87 (1 H, s, 3-H), 6.35 (1 H, s, 5-H), $7.23(2 \mathrm{H}, \mathrm{m}), 7.39$ (1 $\mathrm{H}, \mathrm{m})$ and $7.45(2 \mathrm{H}, \mathrm{m}) ; \delta_{\mathrm{C}}\left(\mathrm{CDCl}_{3} ; 75 \mathrm{MHz}\right) 42.6(\mathrm{q}, \mathrm{NMe}), 57.1$ (q, OMe), 97.8 (d, C-3), 100.0 (d, C-5), 112.2 (s, CN), 112.3 (s, CN), 113.1 (s, CN), 113.9 (s, CN), 125.8 (s, C-6), 125.9 (d, 2 C, C$\left.2^{\prime}, C_{-6}\right), 129.0$ (d, C-4'), 130.2 (d, 2 C, C-3', C-5'), 130.6 (s, C-2), $134.8\left(\mathrm{~s}, \mathrm{C}-1^{\prime}\right), 143.3(\mathrm{~s}, \mathrm{C}-1)$ and $169.0(\mathrm{~s}, \mathrm{C}-4) ; \mathrm{m} / z 315\left(\mathrm{M}^{+}\right.$, 46\%), 314 (100), $300(23), 213(20), 106(35)$ and $77(97)\left(\mathrm{M}^{+}\right.$, 315.1109. $M, 315.1120)$.

Dimethyl 6-Cyano-4-methoxy-6-(N-methylanilino)hexa-1,3,5triene-2,3-dicarboxylate 11.-A benzene solution $\left(5 \mathrm{~cm}^{3}\right)$ of the diene $1(E / Z$ mixture, $120 \mathrm{mg}, 0.56 \mathrm{mmol})$ and dimethyl acetylenedicarboxylate $(80 \mathrm{mg}, 0.56 \mathrm{mmol})$ was refluxed at $80^{\circ} \mathrm{C}$ for $16 \mathrm{~h}$. The solvent was removed, and the residue was chromatographed on $\mathrm{SiO}_{2}$ by elution with gradients of EtOAc in hexane to give compound $11(175 \mathrm{mg}, 88 \%)$, as a mixture of $(3 E, 5 Z)$ - and $(3 E, 5 E)$-isomers $(3: 1)$. The analytical sample of the $Z$-isomer was obtained by HPLC, eluting with $2 \%$ EtOAc in hexane.

$Z$-Isomer: yellow oil; $v_{\max }$ (neat) $/ \mathrm{cm}^{-1} 2947,2840,2227$, $1721,1569,1431,1077,994,879$ and $766 ; \delta_{\mathrm{H}}\left(\mathrm{CDCl}_{3} ; 300\right.$ MHz) 3.32 (3 H, s, NMe), 3.63 (3 H, s, OMe), 3.67 (3 H, s, $\mathrm{OMe}), 3.74(3 \mathrm{H}, \mathrm{s}, \mathrm{OMe}), 5.74(1 \mathrm{H}, \mathrm{d}, J 1.2), 6.46(1 \mathrm{H}, \mathrm{d}, J 1.2$ $\mathrm{Hz}), 6.80(1 \mathrm{H}, \mathrm{s}, 5-\mathrm{H}), 7.24(3 \mathrm{H}, \mathrm{m})$ and $7.36(2 \mathrm{H}, \mathrm{m}) ; \delta_{\mathrm{C}^{-}}$ $\left(\mathrm{CDCl}_{3} ; 75 \mathrm{MHz}\right) 41.5$ (q, NMe), 51.7 (q, OMe), 52.2 (q, OMe), 60.2 (q, OMe), 110.4 (d, C-5), 114.1 (s, CN), 124.5 (s, C-6), 125.1 (s, C-3), 125.4 (d, 2 C, C-2', C-6'), 125.7 (s, C-2), 126.8 (d, C-4'), $129.2\left(\mathrm{t},=\mathrm{CH}_{2}\right), 129.5\left(\mathrm{~d}, 2 \mathrm{C}, \mathrm{C}-3^{\prime}, \mathrm{C}-5^{\prime}\right), 135.6\left(\mathrm{~s}, \mathrm{C}-1^{\prime}\right), 145.1$, (s, $\mathrm{C}-4), 164.4(\mathrm{~s}, \mathrm{C}=\mathrm{O})$ and $167.3(\mathrm{~s}, \mathrm{C}=\mathrm{O}) ; \mathrm{m} / \mathrm{z} 356\left(\mathrm{M}^{+}, 67 \%\right), 355$ (100), $341(18), 297$ (93), $265(51), 237$ (30), $169(20)$ and 77 (97) $\left(\mathrm{M}^{+}\right.$, 356.1366. $\left.M, 356.1372\right)$.

$E$-Isomer (mixed with the $Z$-isomer): yellow oil; $v_{\max }$ (neat) $/ \mathrm{cm}^{-1} 2947,2840,2222(\mathrm{CN}), 1722,1574,1490,1130$, 1077,991 and $759 ; \delta_{\mathrm{H}}\left(\mathrm{CDCl}_{3} ; 300 \mathrm{MHz}\right) 3.30(3 \mathrm{H}, \mathrm{s}, \mathrm{NMe})$, 3.59 ( $3 \mathrm{H}, \mathrm{s}, \mathrm{OMe}), 3.68$ (3 H, s, OMe), 3.69 (3 H, s, OMe), 5.74 $(1 \mathrm{H}, \mathrm{d}, J 1.6), 5.85(1 \mathrm{H}, \mathrm{s}, 5-\mathrm{H}), 6.52(1 \mathrm{H}, \mathrm{d}, J 1.6), 7.00(2 \mathrm{H}$, $\mathrm{m}), 7.18(1 \mathrm{H}, \mathrm{m})$ and $7.34(2 \mathrm{H}, \mathrm{m}) ; \delta_{\mathrm{C}}\left(\mathrm{CDCl}_{3} ; 75 \mathrm{MHz}\right) 40.1$ (q, NMe), 52.0 (q, OMe), 52.4 (q, OMe), 59.1 (q, OMe), 117.0 (d, C-5), 118.1 (s, CN), 118.9 (d, 2 C, C-2', C-6'), 123.6 (d, C-4'), 123.8 (s, C-3), 125.5 (s, C-2), $129.3\left(\mathrm{t},=\mathrm{CH}_{2}\right), 131.8$ (d, $2 \mathrm{C}, \mathrm{C}-3^{\prime}$, C-5'), $145.1\left(\mathrm{~s}, \mathrm{C}-1^{\prime}\right)$ and $158.2(\mathrm{~s}, \mathrm{C}-4)$.

Ethyl 4-Cyano-2-(N-methylanilino)benzoate 12a and Ethyl 2Cyano-4-(N-methylanilino)benzoate $12 \mathrm{~b}$. - A toluene solution $\left(5 \mathrm{~cm}^{3}\right)$ of the diene $1(E / Z$ mixture, $167 \mathrm{mg}, 0.78 \mathrm{mmol})$ and ethyl propiolate $\left(0.08 \mathrm{~cm}^{3}, 0.86 \mathrm{mmol}\right)$ was refluxed at $110^{\circ} \mathrm{C}$ in a sealed tube for $16 \mathrm{~h}$. The solvent was removed, and the residue was chromatographed on $\mathrm{SiO}_{2}$ by elution with $2 \%$ EtOAc in hexane to give 12a $(53 \mathrm{mg})$ followed by a mixture of $12 \mathrm{a}, \mathbf{b}(3: 1$, $80 \mathrm{mg}$ ).

Isomer 12a: Yellow oil; $v_{\max }($ neat $) / \mathrm{cm}^{-1} 2981,2227(\mathrm{CN})$, $1705(\mathrm{C}=\mathrm{O}), 1581,1490,1361$ and $1270 ; \delta_{\mathrm{H}}\left(\mathrm{CDCl}_{3} ; 300\right.$ $\mathrm{MHz}) 1.38$ (3 H, t, J 7.2), 3.35 (3 H, s, NMe), 4.37 ( $2 \mathrm{H}, \mathrm{q}, J$ 7.2), $6.84(1 \mathrm{H}, \mathrm{dd}, J 8.8,2.6,5-\mathrm{H}), 6.98(1 \mathrm{H}, \mathrm{d}, J 2.6,3-\mathrm{H}), 7.19(2 \mathrm{H}$, $\mathrm{m}), 7.27(1 \mathrm{H}, \mathrm{m}), 7.43(2 \mathrm{H}, \mathrm{m})$ and $7.90(1 \mathrm{H}, \mathrm{d}, J 8.8,6-\mathrm{H}) ; \delta_{\mathrm{C}^{-}}$ $\left(\mathrm{CDCl}_{3} ; 75 \mathrm{MHz}\right) 14.2$ (q, Me), 40.2 (q, NMe), $61.3\left(\mathrm{t}, \mathrm{OCH}_{2}\right)$, 114.0 (s, CN), 115.9 (d, C-3), 118.3 (s, C-4), 119.0 (d, C-4'), 119.8 (s, C-1), 126.5 (d, 2 C, C-2', C-6'), 126.8 (d, C-5), 130.3 (d, 2 C, C-3', C-5'), 132.5 (d, C-6), 146.1 (s, C-1'), 151.7 (s, C-2) and 
$164.3(\mathrm{~s}, \mathrm{C}=\mathrm{O}) ; \mathrm{m} / z 280\left(\mathrm{M}^{+}, 85 \%\right), 175(14), 160(32), 132(100)$, $106(62)$ and $77(32) .\left(\mathrm{M}^{+} 280.1210 . M, 280.1212\right)$.

Isomer 12b (mixed with 12a): $\delta_{\mathrm{H}}\left(\mathrm{CDCl}_{3} ; 300 \mathrm{MHz}\right) 1.40(3$ $\mathrm{H}, \mathrm{t}, J 7.2 \mathrm{~Hz}), 3.87(3 \mathrm{H}, \mathrm{s}, \mathrm{NMe}), 4.40(2 \mathrm{H}, \mathrm{q}, J 7.2), 7.10(1 \mathrm{H}$, $\mathrm{dd}, J 9.3,2.8), 7.22(4 \mathrm{H}, \mathrm{m}), 7.43(2 \mathrm{H}, \mathrm{m})$ and $8.06(1 \mathrm{H}, \mathrm{d}, J 9.3)$; $\delta_{\mathrm{C}}\left(\mathrm{CDCl}_{3}\right) 14.1(\mathrm{q}, \mathrm{Me}), 55.9(\mathrm{q}, \mathrm{NMe}), 61.8\left(\mathrm{t}, \mathrm{OCH}_{2}\right), 114.0$ (s, CN), 118.3 (s, C-2), 119.0 (C-4'), 119.8 (s, C-1), 119.9 (d, C-3, C-5), 126.5 (d, 2 C, C-2', C-6'), 130.3 (d, 2 C, C-3', C-5'), 133.1 (d, $\mathrm{C}-6), 146.1\left(\mathrm{~s}, \mathrm{C}-1^{\prime}\right), 158.5(\mathrm{~s}, \mathrm{C}-4)$ and $162.6(\mathrm{~s}, \mathrm{C}=\mathrm{O})$.

\section{Acknowledgements}

We thank the National Science Council of the Republic of China for financial support of this work.

\section{References}

1 (a) H. Ahlbrecht and K. Pfaff, Synthesis, 1985, 421; (b) H. Ahlbrech and M. Ibe, Synthesis, 1988, 210; (c) J. M. Fang and H. T. Chang, J. Chem. Soc., Perkin Trans. 1, 1988, 1945.

2 (a) J. L. Boucher and L. Stella, Tetrahedron, 1985, 41, 875; (b) D. Dopp, H. R. Mamarian, C. Kruger and E. Rabbe, Chem. Ber., 1989 122,585 .

3 (a) J. M. Fang and C. C. Yang, J. Chem. Soc., Chem. Commun., 1985, 1356; (b) J. M. Fang, C. C. Yang and Y. W. Wang, J. Org. Chem., $1989,54,477$
4 F. Mohamadi and W. C. Still, Tetrahedron Lett., 1986, 27, 893.

5 (a) R. Gompper, Angew. Chem., Int. Ed. Engl., 1969, 8, 312; (b) M. Petrzilka and J. I. Grayson, Synthesis, 1981, 753; (c) P. W. Hickmott, Tetrahedron, 1982, 38, 3363; (d) P. W. Hickmott, Tetrahedron, 1984, 40, 2989.

6 (a) J. K. Rasmussen and A. Hassner, Chem. Rev., 1976, 76, 389; (b) J. R. Malpass and N. J. Tweddle, J. Chem. Soc., Perkin Trans. 1, 1977, 874

7 (a) N. S. Isaacs, J. Chem. Soc. B, 1966, 1053; (b) Z. Rappoport, J. Chem. Soc., 1963, 4498.

8 (a) R. Huisgen, Acc. Chem. Res., 1977, 10, 117; (b) R. Huisgen, Acc. Chem. Res., 1977, 10, 199.

9 J. Froborg and G. J. Magnusson, J. Am. Chem. Soc., 1978, 100, 6728.

10 E. Pretsch, J. Seibl, W. Simon and T. Clerc, Table of Spectral Data for Structure Determination of Organic Compounds, Springer-Verlag, Berlin, 1983, pp. C120-C125, H255-H260.

11 (a) C. W. Spangler, B. Keys and D. C. Bookbinder, J. Chem. Soc. Perkin Trans. 2, 1979, 810; (b) H. Y. Elnagar and W. H. Okamura, $J$. Org. Chem., 1988, 53, 3060.

12 J. H. Boyer and J. Kooi, J. Am. Chem. Soc., 1976, 98, 1099.

13 G. A. Russell and M. Ballenegger, Synthesis, 1973, 104

Paper 2/00611A

Received 4th February 1992 Accepted 18th February 1992 